\title{
Region-Based Artificial Terrain Texture Generation
}

\author{
Qicheng $\mathrm{Li}^{1}$, Chen Zhao ${ }^{2}$, Qiang Zhang ${ }^{2}$,Weining Yue ${ }^{1}$, and Guoping Wang ${ }^{1}$ \\ ${ }^{1}$ Dep. of Computer Science \& Technology, Peking University, Beijing, China \\ \{lqc, yue, wgp \} @graphics.pku.edu.cn \\ ${ }^{2}$ Service Experience Research, IBM China Research Laboratory, Beijing, China \\ \{zhaochen, zhqiang\} $\varrho_{\mathrm{cn}}$. ibm. com
}

\begin{abstract}
Terrain texture plays a great role on visualization in many 3D applications. Sometimes terrain texture is difficult to obtain in a desired area, thus a new approach to terrain texture generation based on satellite images is proposed to resolve this problem. We prepare a silhouette map and a satellite image for the desired terrain texture and colorize the two pictures with the same pattern according to the terrain features. Then a method of texture synthesis is adopted to generate the final terrain texture.
\end{abstract}

\section{Introduction}

For a long time research in terrain visualization has been concerned with developing techniques to cope with complex terrain geometries. While LOD terrain models as well as image based modeling and rendering techniques form the basis of any kind of large-scale, real-time terrain visualization, texturing techniques for terrain visualization have not been studied as much.

Terrain-texturing refers to the method used to fill the polygons that comprise the terrain database. The three primary texturing concepts tested were constant-color (CC), elevation-based generic (EBG), and photo-realistic (PR). The CC texturing concept represented an industry concept that has completed the process of Federal Aviation Administration (FAA) certification in the Capstone-program. The EBG texturing concept consisted of twelve equal-height coloring bands that correspond to different absolute terrain elevation levels, similar to the colors employed for Visual Flight Rules (VFR) sectional charts. The PR texturing concept was created by overlaying color ortho-rectified satellite imagery data onto a DEM database. And our terrain texturing method belongs to the third category.

Recently texture synthesis from samples has become a novel technology of texture generation. Compared with traditional texture mapping, texture synthesis from samples can avoid gaps and distortion. So we adopt texture synthesis from satellite imagery to generate the terrain texture.

\section{Related Work}

Texture synthesis has been an active area of research for many years. Numerous approaches have been proposed for texture analysis and synthesis. Here we briefly review some recent and representative works. 


\subsection{Physical Simulation}

One way to synthesize textures is to directly simulate their physical generation processes. Biological patterns such as fur, scales, and skin can be modeled using reaction diffusion [1] and cellular texturing [2]. These techniques can produce textures directly on 3D meshes so distortion problem in texture mapping is avoided. However, different textures are usually generated by different physical processes so these approaches are applicable to only a limited class of textures.

\subsection{Markov Random Field}

Many algorithms model textures by Markov Random Fields and generate textures by probability sampling [3]. Since Markov Random Fields have been proven to be a good approximation for a broad rang of textures, these algorithms are general and some of them produce good results. A drawback of Markov Random Field sampling, though, is that it is computationally expensive: even small texture patches can take hours or days to generate

\subsection{Feature Matching}

Some algorithms model textures as a set of features, and generate new images by matching the features in an example textures. Heeger and Bergen [4] introduced this problem to the computer graphics community in 1995. More recently, De Bonet and Efros and Leung [5] showed that a nearest-neighbor search can perform high-quality texture synthesis in a single pass, using multiscale and single-scale neighborhoods, respectively. Wei and Levoy [6] unify these approaches, using neighborhoods consisting of pixels both at the same scale and at coarser scales.

In this paper, we aim to introduce a texture synthesis based on feature matching approach to 3D terrain visualization. Our method can generate discretional terrain texture through simple interaction. This is a continued work of our terrain generation [7].

\section{Terrain Texture Generation}

The integrated terrain model requires both the geometrical data and upper information. The realistic upper information is the terrain color (texture) usually viewed from a vertical angle as those in aerial or satellite images. Such imagery, rare just ten years ago, is now widely available from commercial remote sensing agencies, such as Spot and Landsat. The aerial or satellite images are good sources to generate terrain texture. But the whole satellite image is not a Markov random field and we can not generate a terrain texture synthesis by sampling. However, various sub-regions of satellite images (such as forest, river, and plain) are Markov random fields, so we propose a regionbased realistic terrain texture generation. Figure 1 is an example of satellite images.

\subsection{Satellite Image Segment}

This phase is to divide the satellite image into different regions and colorize the corresponding terrain type in the image. The colors selected to fill the image should be 


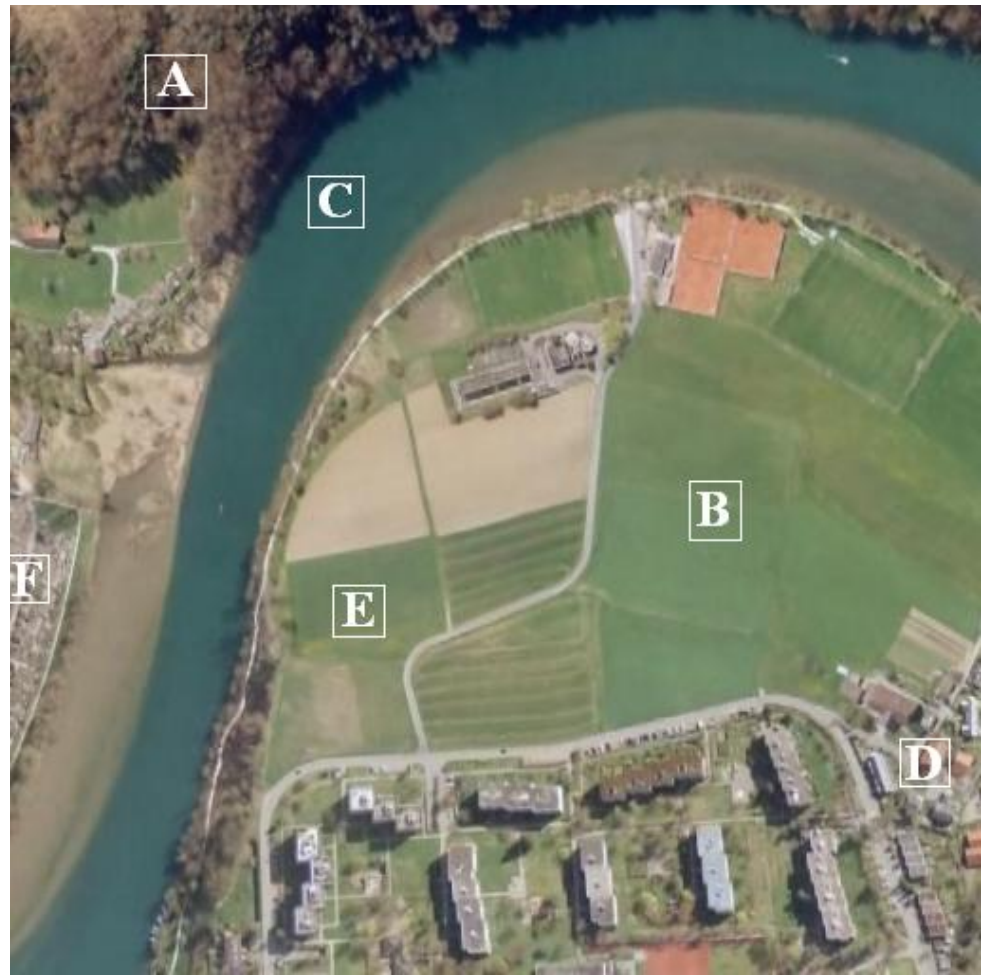

Fig. 1. A satellite image. The region A, B, C, D, E, F are the texture feature areas.
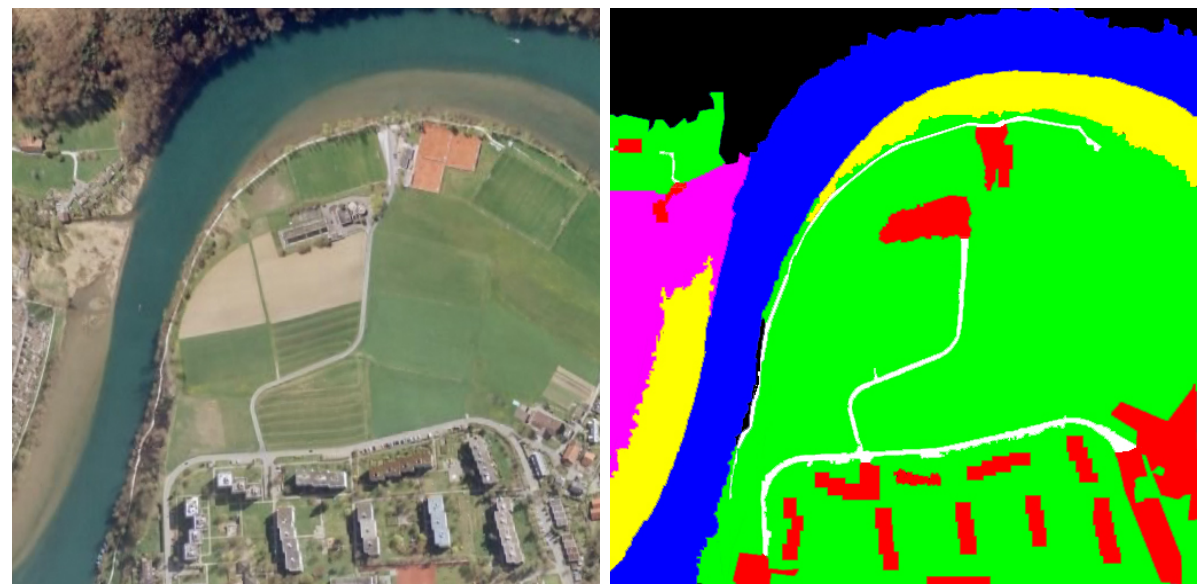

Fig. 2. The image colorized process 


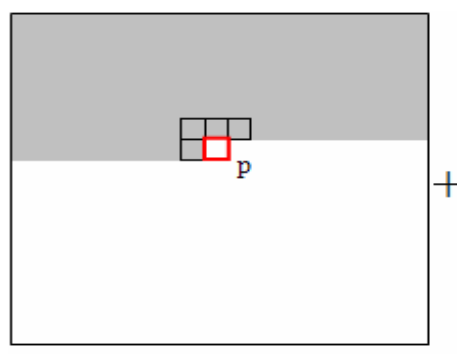

(a)

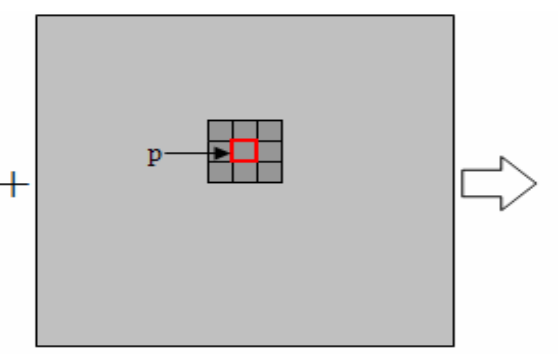

(b)

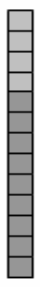

(c)

Fig. 3. The pixel feature vector set $\Psi$, (a) the $\boldsymbol{L}$-neighbor pixels in the satellite image, (b) the 8 -neighbor pixels in the colored image, (c) the pixel feature vector

recognized by computer easily, so we select the ultimate colors of RGB-space such as red $(255,0,0)$, blue $(0,0,255)$ and yellow $(255,255,0)$. Because the content of the satellite image is very complex, none of image processing software can segment the image automatically. And we use Adobe-PhotoShop to colorize the image on hand (Figure 2).

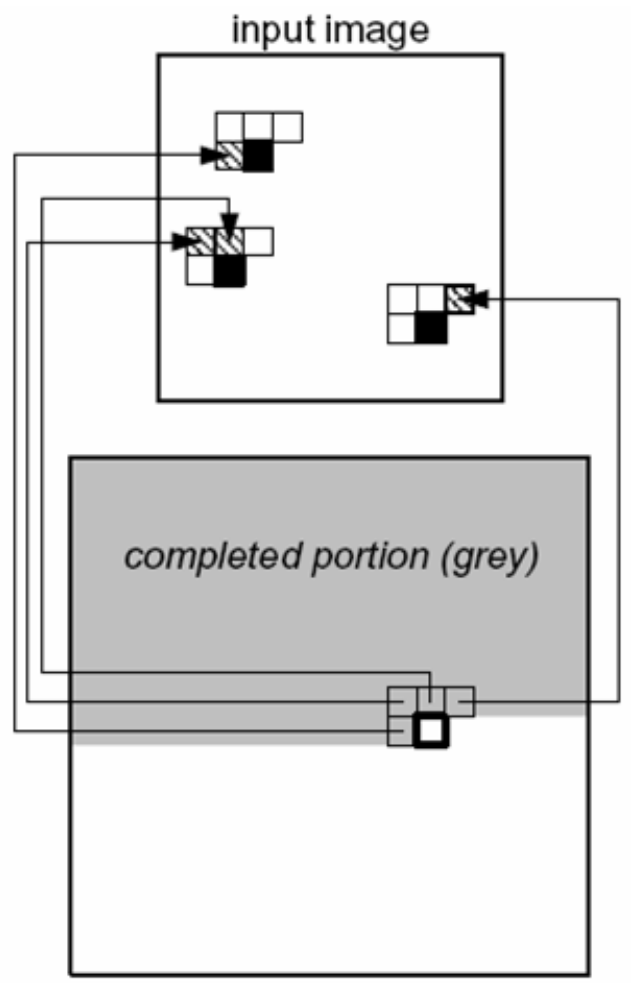

Fig. 4. Ashikhmin's approach 


\subsection{Texture Synthesis}

For generating the desired terrain texture, we must prepare a terrain silhouette map. The terrain silhouette map is divided into several child-regions according to different terrain features. And we colorize the terrain silhouette map to get a colored terrain map. Now we have a satellite image $\boldsymbol{A}^{\prime}$, the colored image $\boldsymbol{A}$ and the colored terrain map $\boldsymbol{B}$. Finally we synthesize the generated texture $\boldsymbol{B}^{\prime}$ from the satellite image $\boldsymbol{A}^{\prime}$, through which we choose the appropriate parts of the transform $\boldsymbol{A} \rightarrow \boldsymbol{A}^{\prime}$ in synthesizing $\boldsymbol{B} \rightarrow \boldsymbol{B}$. The texture synthesis is implemented as follows:

1. Build the pixel feature vector set $\Psi$ of the satellite image $\boldsymbol{A}^{\prime}$, the elements of which are the RGB values of the $\boldsymbol{L}$-neighbor pixels in the satellite image and the 8-neighbor pixels in the colored image (Figure 3).

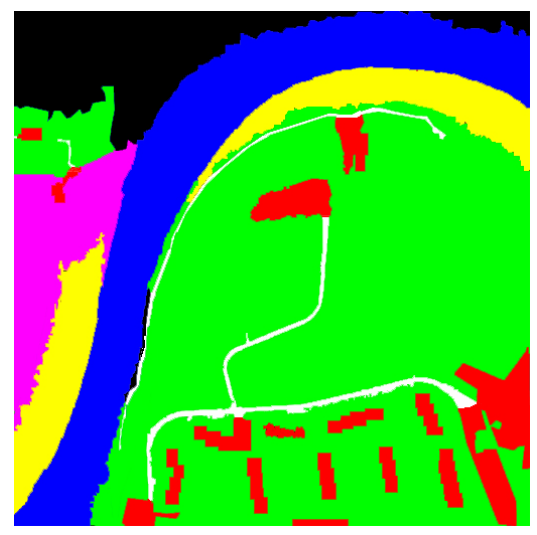

A: the colored image

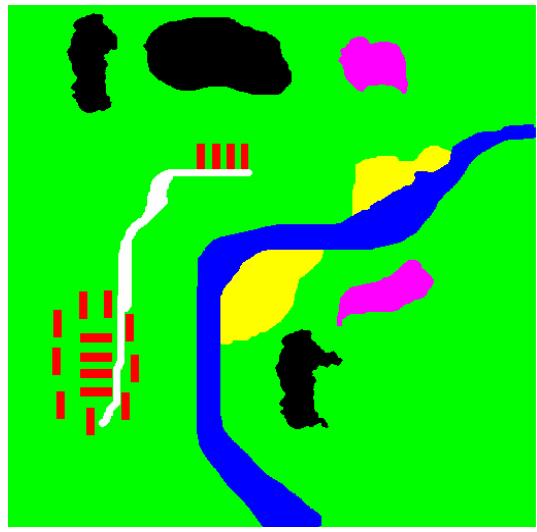

B: the colored terrain map

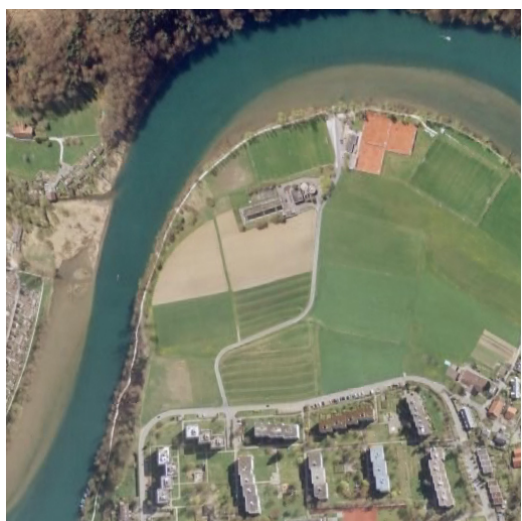

A': the satellite image

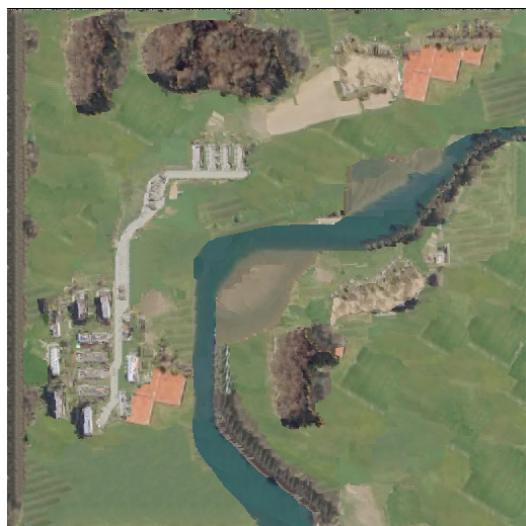

B': the generated texture

Fig. 5. The texture generation 
2. Synthesize the generated texture $\boldsymbol{B}^{\prime}$ in scan-line order. For each pending pixel $\boldsymbol{p}$ :

a) Compute the feature vector $v_{p}$ of the pixel $\boldsymbol{p}$ according to colored terrain map $\boldsymbol{B}$ and the texture $\boldsymbol{B}$ ' which has the established pixels in scan-line.

b) Find the most ANN-suited pixel feature vector $v_{\text {best-ann }}$ from the set $\Psi$ by an approximate-nearest-neighbor search (ANN) [8] and get the most suited pixel $p_{\text {best-ann }}$.

c) Find the most coherence-suited pixel $p_{\text {best-coherence }}$ according to a coherence search, based on Ashikhmin's approach [9].

3. Compare the $p_{\text {best-ann }}$ and the $p_{\text {best-coherence }}$, find out the best pixel $p_{\text {best }}$, and set the color of the best pixel $p_{\text {best }}$ to the pending pixel $\boldsymbol{p}$.

Through the aforementioned texture synthesis process, we can get an artificial terrain texture (Figure 5) which well matches the terrain region.

\section{Conclusion and Future Work}

We have presented a new approach for terrain texture generation based on regions. A method of texture synthesis from samples is used to create the terrain texture, and our approach adapts to the complex terrain texture generation. Since the satellite image is the resource of our texture synthesis, the generated texture is authentic and realistic.

There is still much room for improvement. First, the colored satellite image is created interactively, thus designing an automatic method is our next work. Secondly, the texture synthesis is a time-consuming process, especially when generating a huge terrain texture. Improving the efficiency is also our next step.

Acknowledgments. This study was supported by China 973 Program Grant No.2004CB719403, China 863 Program Grant No.2006AA01Z324075, and China National Natural Science Foundation No. 60473100.

\section{References}

1. Witkin, A., Kass, M.: Reaction-diffusion textures. In: Sederberg, T. W.(ed.) Computer Graphics (SIGGRAPH'91 Proceedings), vol. 25, pp. 299-308 (1991)

2. Worley, S.P.: A cellular texture basis function. In: ACM SIGGRAPH 96 Proceedings, pp. 291-294 (1996)

3. Zhu, S., Wu, Y., Mumford, D.: Filters, random fields and maximun entropy (FRAME) towards a unified theory for texture modeling. International Journal of Computer Vision 27(2), 107-126 (1998)

4. Heeger, D. J., Bergen, J. R.: Pyramid-Based Texture Analysis/Synthesis. In: ACM SIGGRAPH 95 Proceedings, pp. 229-238 (1995)

5. Efros, A., Leung, T.: Texture synthesis by non-parametric sampling. In: International Conference on Computer Vision '99, vol. 2, pp. 1033-1038 (1999) 
6. Wei, L.-Y., Levoy, M.: Fast Texture Synthesis Using Tree-Structured Vector Quantization. In: ACM SIGGRAPH 00 Proceedings, pp. 479-488 (2000)

7. Li, Q., Wang, G., Zhou, F., Tang, X., Yang, K.: Example-Based Realistic Terrain Generation. In: Pan, Z., Cheok, A., Haller, M., Lau, R.W.H., Saito, H., Liang, R. (eds.) ICAT 2006. LNCS, vol. 4282, pp. 811-818. Springer, Heidelberg (2006)

8. Arya, S., Mount, D.M., Netanyahu, N.S., Silverman, R., Wu, A.Y.: An Optimal Algorithm for Approximate Nearest Neighbor Searching in Fixed Dimensions. Journal of the ACM 45(6), 891-923 (1998)

9. Ashikhmin, M.: Synthesizing natural textures. ACM Symposium on Interactive 3D Graphics, North Carolina, pp. 217-226 (2001) 